USITP-94-15

December-1994

Revised January-1996

\title{
Field theory of anyons in the lowest Landau level
}

\author{
T.H. Hansson ${ }^{\star}$, J.M. Leinaas ${ }^{\dagger}$ and S.Viefers ${ }^{\dagger \$}$
}

\begin{abstract}
We construct a field theory for anyons in the lowest Landau level starting from the $N$ particle description, and discuss the connection to the full field theory of anyons defined using a statistical gauge potential. The theory is transformed to free form, with the fields defined on the circle and satisfying modified commutation relations. The Fock space of the anyons is discussed, and the theory is related to that of edge excitations of an anyon droplet in a harmonic oscillator well.
\end{abstract}

${ }^{\star}$ Fysikum, University of Stockholm, P.O. Box 6730, S-11385 Stockholm, Sweden ${ }^{\dagger}$ Institute of Physics, University of Oslo, P.O. Box 1048 Blindern, N-0316 Oslo, Norway and Centre for Advanced Study, P.O. Box 7606 Skillebekk, 0205 Oslo, Norway ${ }^{\star}$ Supported by the Swedish Natural Science Research Council ${ }^{\$}$ Supported by the Norwegian Research Council 


\section{Introduction}

We consider in this paper the field theoretical formulation of anyons, not in general, but for particles in a strong magnetic field. This is an interesting case, since so far the only physical system where the presence of anyons has been established with any degree of confidence is the quantum Hall system [1]], where the particles move in a strong magnetic field. We assume the field to be sufficiently strong that the particles are constrained to the (generalized) first Landau level. The dynamics of the system is then effectively onedimensional, and this simplifies some of the problems associated with the field theory formulation. For the full anyon problem, i.e. without the restriction to the lowest Landau level, the system can be described either in terms of Bose or Fermi fields with an non-local interaction to take care of the fractional statistics of the particles. Formulations of the theory without this statistics interaction, but with field operators which satisfy modified commutation relations have been attempted [2, 3, 4, 5], but to carry out this consistently has proven to be difficult, since the phase factor associated with particle interchange cannot readily be absorbed in the algebraic properties of the field operators. In the one-dimensional case the problems associated with the ordering and interchange of particles are easier to handle.

To make the one-dimensionality of the anyon system in the lowest Landau level explicit, we map the system onto a circle. It can then be transformed to a free field form, with field operators satisfying modified commutation relations. This leads to unconventional rules for the occupation of single particle levels. We consider a free field form of the Hamiltonian which for the anyon system corresponds to including a harmonic oscillator potential in the Hamiltonian. This field theory takes the form of a chiral Luttinger model, and the formulation is closely related to similar descriptions of edge excitations in the quantum Hall effect [6, 7].

The discussion in the paper is organized as follows. We take, in Section 2, the $N$ particle description of anyons in the lowest Landau level as our starting point, and introduce anyon field operators so that the wave function, in the standard way, can be regarded as the projection of the abstract state vector on the set of position vectors created by the (Hermitian conjugate) field operator. We introduce a transformation to fields with simple commutation relations and express the Hamiltonian and the total angular momentum operator in terms of these field operators. In Section 3, the field theory is transformed to a one-dimensional form with field operators defined on a circle. A further transformation gives a field equation of the free form, but with modified commutation relations. The Fock space representation constructed with these operators is discussed. In the last section we examine the free field theory corresponding to particles in a combined magnetic field and harmonic oscillator potential. This description is similar to the chiral fermion field theory introduced to describe edge excitations in the quantum Hall system. However, in the present case it describes the full dynamics of the system of (non-interacting) particles in

the lowest Landau level. We also show that neglecting finite size effects, it is equivalent to the description given by Wen [6] of the edge of the quantum Hall state with filling fraction $1 /(2 m+1)$. In Appendix A we derive the field operators in the lowest Landau level from 
the (bosonic) field operators of the full anyon system. In Appendix B we give a Lagrangian formulation for the corresponding system of particles on the circle, and in Appendix $\mathrm{C}$ we give a summary of the statistical mechanics for this system.

\section{Field operators in the lowest Landau level}

The general form of a multivalued $N$-anyon wave function in the lowest Landau level is

$$
\Psi(z, \bar{z})=\prod_{i<j}\left(z_{i}-z_{j}\right)^{\nu} \psi(z) e^{-\frac{1}{2} \sum_{i} \bar{z}_{i} z_{i}},
$$

where dimensionless complex coordinates have been used,

$$
z=\sqrt{\frac{e B}{2}}(x+i y) .
$$

$B$ is here the magnetic field and $(x, y)$ are the coordinates of the two-dimensional plane where the particles are moving. We use $z$ also as a shorthand notation for the set of coordinates, $z_{1}, \ldots, z_{N}$, of the $N$-particle system. The holomorphic part of the wavefunction, $\psi(z)$, is either a fully symmetric, or a fully antisymmetric function of the particle coordinates. We refer to these two possibilities as the bosonic and fermionic representations respectively. $\nu$ is the statistics parameter of the anyons, which is 0 for bosons and 1 for fermions in the bosonic representation. In this section and in Appendix A and B we mainly use this representation, whereas both possibilities are included explicitly when the field theory on the circle is introduced in Section 3. In the discussion related to the anyon droplet (Section 4 and Appendix C) however, the fermionic representation is the natural one to choose.

We introduce a basis of position vectors in the lowest Landau level through the analytic part of the wave function in the following way

$$
\psi(z)=\langle z \mid \psi\rangle
$$

The field operator $\phi(z)$ is defined by

$$
\phi(z)|0\rangle=0
$$

and

$$
|\bar{z}\rangle=\left|\bar{z}_{1}, \ldots \bar{z}_{N}\right\rangle=\frac{1}{\sqrt{N !}} \phi^{\dagger}\left(\bar{z}_{1}\right) \ldots \phi^{\dagger}\left(\bar{z}_{N}\right)|0\rangle
$$

with $|0\rangle$ as the vacuum state and $\phi^{\dagger}(\bar{z})$ as the Hermitian conjugate field operator. This definition gives the standard expression for the wave function $\psi(z)$ in terms of the field operator,

$$
\psi(z)=\psi\left(z_{1}, \ldots z_{N}\right)=\frac{1}{\sqrt{N !}}\left\langle 0\left|\phi\left(z_{N}\right) \ldots \phi\left(z_{1}\right)\right| \psi\right\rangle
$$


The field operators act within the lowest Landau level, and the action of the operators on vectors orthogonal to this subspace is defined as zero.

Consider the scalar product between two states of the lowest Landau level,

$$
\begin{aligned}
\left\langle\psi \mid \psi^{\prime}\right\rangle & =\int d^{2} z_{1} \ldots d^{2} z_{N} \prod_{i<j}\left|z_{i}-z_{j}\right|^{2 \nu} e^{-\sum_{i} \bar{z}_{i} z_{i}} \bar{\psi}(\bar{z}) \psi^{\prime}(z) \\
& =\left\langle\psi\left|\int d^{2} z_{1} \ldots d^{2} z_{N} e^{-\sum_{i} \bar{z}_{i} z_{i}} \gamma_{N}(z, \bar{z})\right| \bar{z}_{1} \ldots . \bar{z}_{N}\right\rangle\left\langle z_{1} \ldots z_{N} \mid \psi^{\prime}\right\rangle
\end{aligned}
$$

where

$$
\gamma_{N}(z, \bar{z})=\prod_{i<j}\left|z_{i}-z_{j}\right|^{2 \nu}
$$

This gives the completeness relation,

$$
\int d^{2} z_{1} \ldots d^{2} z_{N} e^{-\sum_{i} \bar{z}_{i} z_{i}} \gamma_{N}(z, \bar{z})\left|\bar{z}_{1} \ldots . \bar{z}_{N}\right\rangle\left\langle z_{1} \ldots z_{N}\right|=P_{N}
$$

with $P_{N}$ as the projection on the $N$-particle space. This implicitly defines the overlap between basis vectors,

$$
g_{N}\left(z^{\prime}, \bar{z}\right)=\left\langle z^{\prime} \mid \bar{z}\right\rangle
$$

For arbitrary $\psi(z)$ we have

$$
\int d^{2} z_{1} \ldots d^{2} z_{N} e^{-\sum_{i} \bar{z}_{i} z_{i}} \gamma_{N}(z, \bar{z}) g_{N}\left(z^{\prime}, \bar{z}\right) \psi(z)=\psi\left(z^{\prime}\right),
$$

which means that $g_{N}$ acts as a $\delta$-function in the space of analytic functions $\psi(z)$, when the $\nu$-dependent term $\gamma_{N}$ is included in the integration measure. However, because of $\gamma_{N}$ the overlap function $g_{N}$ does not have a simple form. While $g_{1}(z)=e^{z^{\prime} z} / \pi, g_{2}$ is rather complicated,

$$
g_{2}\left(z_{1}^{\prime}, z_{2}^{\prime}, \bar{z}_{1}, \bar{z}_{2}\right)=\frac{1}{2^{\nu} \pi^{2}} e^{\frac{1}{2}\left(z_{1}^{\prime}+z_{2}^{\prime}\right)\left(\bar{z}_{1}+\bar{z}_{2}\right)} \sum_{m=0}^{\infty} \frac{\left[\frac{1}{2}\left(z_{1}^{\prime}-z_{2}^{\prime}\right)\left(\bar{z}_{1}-\bar{z}_{2}\right)\right]^{2 m}}{\Gamma(2 m+\nu+1)} .
$$

When the wave functions $\psi(z)$ are symmetric, the field operators $\phi(z)$ and $\phi\left(z^{\prime}\right)$ commute in spite of the complicated scalar product. However, the scalar product is important for the commutator

$$
\Delta\left(z^{\prime}, \bar{z}\right)=\left[\phi\left(z^{\prime}\right), \phi^{\dagger}(\bar{z})\right]
$$

as is illustrated by the following matrix elements of the operator $\Delta$,

$$
\Delta_{0}\left(z^{\prime}, \bar{z}\right)=\left\langle 0\left|\left[\phi\left(z^{\prime}\right), \phi^{\dagger}(\bar{z})\right]\right| 0\right\rangle=g_{1}\left(z^{\prime}, \bar{z}\right)
$$




$$
\begin{aligned}
\Delta_{1}\left(z_{1}^{\prime}, z_{2}^{\prime}, \bar{z}_{1}, \bar{z}_{2}\right) & =\left\langle z_{1}^{\prime}\left|\left[\phi\left(z_{2}^{\prime}\right), \phi^{\dagger}\left(\bar{z}_{1}\right)\right]\right| \bar{z}_{2}\right\rangle \\
& =2 g_{2}\left(z_{1}^{\prime}, z_{2}^{\prime}, \bar{z}_{1}, \bar{z}_{2}\right)-g_{1}\left(z_{1}^{\prime}, \bar{z}_{1}\right) g_{1}\left(z_{2}^{\prime}, \bar{z}_{2}\right) .
\end{aligned}
$$

Similar, but more complicated, expressions can be found for larger values of the particle number $N$.

That the commutator $\left[\phi\left(z^{\prime}\right), \phi^{\dagger}(\bar{z})\right]$ is a complicated operator and not a simple function of $z^{\prime}$ and $\bar{z}$ implies that the observables, when expressed in terms of $\phi$ and $\phi^{\dagger}$, may take a rather complicated form. This is the case for the particle number as well as for the angular momentum operator, even if these have simple expressions in the $N$-particle formulation. To recover these from the field theory expressions of the operators one has to make use of the commutators between the creation and annihilation operators.

To simplify expressions, we introduce the dual basis $|\bar{z}\rangle\rangle$ by the orthogonality requirement

$$
\left.\left\langle z^{\prime} \mid \bar{z}\right\rangle\right\rangle=\delta_{N}\left(z^{\prime}, \bar{z}\right)
$$

where the $\delta$-function now refers to the integration measure where the $\nu$-dependent factor $\gamma_{N}$ is omitted

$$
\delta_{N}\left(z^{\prime}, \bar{z}\right)=\frac{1}{\pi^{N} N !} \sum_{p e r m\{\bar{z}\}} e^{\sum_{i} z_{i}^{\prime} \bar{z}_{i}} .
$$

The completeness relation then becomes

$$
\begin{aligned}
P_{N} & \left.=\int d^{2} z_{1} \ldots d^{2} z_{N} e^{-\sum_{i} \bar{z}_{i} z_{i}}\left|\bar{z}_{1} \ldots \bar{z}_{N}\right\rangle\right\rangle\left\langle z_{1} \ldots z_{N}\right| \\
& =\int d^{2} z_{1} \ldots d^{2} z_{N} e^{-\sum_{i} \bar{z}_{i} z_{i}}\left|\bar{z}_{1} \ldots \bar{z}_{N}\right\rangle\left\langle\left\langle z_{1} \ldots z_{N}\right|\right.
\end{aligned}
$$

with the two sets of basis vectors related through the expression

$$
|\bar{z}\rangle\rangle=\int d^{2} z_{1}^{\prime} \ldots d^{2} z_{N}^{\prime} e^{-\sum_{i} \bar{z}_{i}^{\prime} z_{i}^{\prime}} \gamma_{N}\left(z^{\prime}, \bar{z}^{\prime}\right) \delta_{N}\left(z^{\prime}, \bar{z}\right)\left|\bar{z}^{\prime}\right\rangle
$$

Corresponding to the new basis we define the dual field operator $\phi^{+}$by the relation

$$
\left.|\bar{z}\rangle\rangle=\left|\bar{z}_{1}, \ldots \bar{z}_{N}\right\rangle\right\rangle=\frac{1}{\sqrt{N !}} \phi^{+}\left(\bar{z}_{1}\right) \ldots \phi^{+}\left(\bar{z}_{N}\right)|0\rangle .
$$

For the operators $\phi$ and $\phi^{+}$we find, in the bosonic representation, the commutation relations

$$
\left[\phi\left(z^{\prime}\right), \phi(z)\right]=\left[\phi^{+}\left(\bar{z}^{\prime}\right), \phi^{+}(\bar{z})\right]=0, \quad\left[\phi\left(z^{\prime}\right), \phi^{+}(\bar{z})\right]=\frac{1}{\pi} e^{z^{\prime} \bar{z}}
$$


These follow from the symmetry of the wave functions and the orthogonality relation (2.16). Due to the simple commutator between $\phi$ and $\phi^{+}$, the basic observables of the system can be given a simple field theoretic form. In the $N$-particle representation the Hamiltonian is simply proportional to the particle number,

$$
H=\frac{e B}{2 m} N
$$

and the angular momentum is

$$
L_{N}=\sum_{i} z_{i} \frac{\partial}{\partial z_{i}}+\frac{\nu}{2} N(N-1) .
$$

In the field theory formulation these operators have the form

$$
H=\frac{e B}{2 m} \int d^{2} z e^{-\bar{z} z} \phi^{+}(\bar{z}) \phi(z)
$$

and

$$
L=\int d^{2} z e^{-\bar{z} z} \phi^{+}(\bar{z})\left(z \frac{\partial}{\partial z}+\frac{\nu}{2} \int d^{2} z^{\prime} e^{-\bar{z}^{\prime} z^{\prime}} \phi^{+}\left(\bar{z}^{\prime}\right) \phi\left(z^{\prime}\right)\right) \phi(z),
$$

as can readily be verified by applying them to an $N$-particle wave funtion $\langle z \mid \psi\rangle$.

We stress that the dual field operator $\phi^{+}$is different from the Hermitian conjugate operator $\phi^{\dagger}$. As already discussed, the observables (2.25) and (2.26) cannot be written in a simple form in terms of the field operators $\phi$ and $\phi^{\dagger}$. If we formally write the relation (2.20) between the two sets of basis vectors as

$$
|\bar{z}\rangle\rangle=\Lambda|\bar{z}\rangle
$$

the operators $\phi^{+}$and $\phi^{\dagger}$ are related by

$$
\phi^{+}=\Lambda \phi^{\dagger} \Lambda^{-1}
$$

The operator $\Lambda$ is positive definite and Hermitian, so it can be written in the form

$$
\Lambda^{-1}=S^{\dagger} S
$$

where $S$ is a non-singular operator. This operator, which is only partly specified by its relation to $\Lambda$, can be used to introduce a new set of field operators, by the similarity transformation

$$
\varphi(z)=S \phi(z) S^{-1} \quad \varphi^{\dagger}(\bar{z})=S \phi^{+}(\bar{z}) S^{-1}
$$

For the new field operators we have the same simple commutation relations (2.22) as for $\phi$ and $\phi^{+}$. Also the transformed observables have the same form as in (2.25) and (2.26), but 
with $\phi$ and $\phi^{+}$replaced by $\varphi$ and $\varphi^{\dagger}$. In this way one can obtain a more standard form of the observables in terms of the field operator and its Hermitian conjugate, but for the transformation (2.30) we do not have an explicit expression.

In this section we have introduced field operators starting from the $N$-particle operators constrained to the lowest Landau level. In Appendix A we show how to relate this description to the field theory description of the full anyon system, with the fractional statistics appearing in the form of a non-local interaction.

\section{Field theory on the circle}

The anyon system restricted to the lowest Landau level can be regarded as a onedimensional system. One way to make this explicit is to map the system onto a circle. The field operator $\varphi(z)$ is expanded in angular momentum eigenstates

$$
\varphi(z)=\sum_{l=0}^{\infty} \frac{1}{\sqrt{l !}} \varphi_{l} z^{l}
$$

and the new operators $\chi(\theta)$ are defined as

$$
\chi(\theta)=\sum_{l=0}^{\infty} \frac{1}{\sqrt{2 \pi}} \varphi_{l} e^{i l \theta} .
$$

In the following we include in our discussion both the bosonic and fermionic anyonrepresentations. In the fermionic case the commutators (2.22) are changed to anticommutators, and with both possibilities included in one equation, the commutator/anticommutator of the fields $\chi$ is

$$
\left[\chi\left(\theta^{\prime}\right), \chi^{\dagger}(\theta)\right]_{ \pm}=\frac{1}{2 \pi} \sum_{l=0}^{\infty} e^{i l\left(\theta^{\prime}-\theta\right)} \equiv \delta_{p e r}^{+}\left(\theta^{\prime}-\theta\right),
$$

where $\delta_{p e r}^{+}(\theta)$ is the positive frequency part of the periodic delta function. The angular momentum has the following form in terms of the new operators

$$
H_{L} \equiv L=\int_{0}^{2 \pi} d \theta \chi^{\dagger}(\theta)\left[-i \partial_{\theta}+\frac{\nu}{2} \int_{0}^{2 \pi} d \theta^{\prime} \chi^{\dagger}\left(\theta^{\prime}\right) \chi\left(\theta^{\prime}\right)\right] \chi(\theta)
$$

We shall in this section consider $L$ to be the Hamiltonian of the system, and hereafter refer to it as $H_{L}$. This essentially corresponds to introducing a harmonic oscillator potential in addition to the magnetic field, as we discuss further in the next section. The equation of motion becomes

$$
\partial_{t} \chi(\theta)=i[L, \chi(\theta)]=-\left(\partial_{\theta}+i \nu N\right) \chi(\theta)
$$

where $N$ is the particle number operator. The $\nu$-dependent "statistics" term can be eliminated by the following transformation:

$$
\chi(\theta) \rightarrow \psi(\theta)=e^{i \nu \theta N} \chi(\theta), \quad N=\int_{0}^{2 \pi} d \theta \psi^{\dagger}(\theta) \psi(\theta)
$$


and the new field operator satisfies the field equation

$$
\left(\partial_{t}+\partial_{\theta}\right) \psi(\theta)=0
$$

which has the form of a free field theory of massless, chiral fermions on the circle. The new fields obey modified commutation relations,

$$
\begin{aligned}
\psi(\theta) \psi\left(\theta^{\prime}\right) \pm e^{-i \nu\left(\theta-\theta^{\prime}\right)} \psi\left(\theta^{\prime}\right) \psi(\theta) & =0 \\
\psi^{\dagger}(\theta) \psi^{\dagger}\left(\theta^{\prime}\right) \pm e^{-i \nu\left(\theta-\theta^{\prime}\right)} \psi^{\dagger}\left(\theta^{\prime}\right) \psi^{\dagger}(\theta) & =0 \\
\psi(\theta) \psi^{\dagger}\left(\theta^{\prime}\right) \pm e^{i \nu\left(\theta-\theta^{\prime}\right)} \psi^{\dagger}\left(\theta^{\prime}\right) \psi(\theta) & =\Delta\left(\theta-\theta^{\prime}\right)
\end{aligned}
$$

with + and - referring to the fermionic and bosonic representations, respectively. The operator $\Delta(\theta)$ is defined as

$$
\begin{aligned}
\Delta(\theta) & =e^{i \nu N \theta} \delta_{p e r}^{+}(\theta) \\
& =\frac{1}{2 \pi} \sum_{l=0}^{\infty} e^{i(l+\nu N) \theta}
\end{aligned}
$$

and is the natural modification of the $\delta$-function defined in (3.3), in the sense that

$$
\begin{aligned}
\int_{0}^{2 \pi} d \theta^{\prime} \psi^{\dagger}\left(\theta^{\prime}\right) \Delta\left(\theta^{\prime}-\theta\right) & =\psi^{\dagger}(\theta) \\
\int_{0}^{2 \pi} d \theta^{\prime} \Delta\left(\theta-\theta^{\prime}\right) \psi\left(\theta^{\prime}\right) & =\psi(\theta) .
\end{aligned}
$$

The commutation relations given above are similar, but not identical, to generalized commutation relations that previously have been considered for one-dimensional theories. (See for example Chap. 7 in [8].)

We note that, due to the unconventional commutation relations satisfied by the field operators, the angular momentum does not have the free field form, but is instead given by

$$
H_{L}=\int_{0}^{2 \pi} d \theta \psi^{\dagger}(\theta)\left[-i \partial_{\theta}-\frac{\nu}{2} \int_{0}^{2 \pi} d \theta^{\prime} \psi^{\dagger}\left(\theta^{\prime}\right) \psi\left(\theta^{\prime}\right)\right] \psi(\theta) .
$$

The interaction term conspires with the unconventional commutation relations to give the free field equation of motion (3.7) for $\psi(\theta)$.

The discussion in this section is entirely in a Hamiltonian framework, but there should also be a Lagrangian formulation, which would be appropriate for a path integral approach. In Appendix B we discuss the classical Lagrangian formulation, and show how to define Poisson brackets which, upon quantization, reproduce the commutation relations (3.8) to first order in $\nu$.

Now consider the Fourier transform

$$
\psi(\theta)=\sum_{\kappa} \frac{1}{\sqrt{2 \pi}} a_{\kappa} e^{i \kappa \theta} .
$$


In this expansion the Fourier variables are non-integers, but due to the periodicity of the original field $\chi(\theta), \kappa$ is restricted to

$$
\kappa=k+\nu n
$$

where $k$ and $n$ are non-negative integers. If we further introduce a set of basis states

$$
\left|\kappa_{N}, \ldots, \kappa_{1}\right\rangle=\frac{1}{\sqrt{N !}} a_{\kappa_{N}}^{\dagger} \ldots a_{\kappa_{1}}^{\dagger}|0\rangle
$$

we have the restriction

$$
\kappa_{i}=k_{i}+\nu(i-1), \quad i=1, \ldots N .
$$

This means that $a_{\kappa}^{\dagger}$ annihilates all states where the integer $n$ is not equal to the particle number of the state. In operator form this is expressed through the identity

$$
a_{\kappa}^{\dagger} e^{-2 \pi i \nu N}=e^{-2 \pi i \kappa} a_{\kappa}^{\dagger}, \quad N=\sum_{\kappa} a_{\kappa}^{\dagger} a_{\kappa} .
$$

The operators $a_{\kappa}$ and $a_{\kappa}^{\dagger}$ satisfy the unconventional commutation relations

$$
\begin{array}{r}
a_{\kappa}^{\dagger} a_{\lambda}^{\dagger} \pm a_{\lambda+\nu}^{\dagger} a_{\kappa-\nu}^{\dagger}=0 \\
a_{\kappa} a_{\lambda} \pm a_{\lambda-\nu} a_{\kappa+\nu}=0 \\
a_{\kappa} a_{\lambda}^{\dagger} \pm a_{\lambda-\nu}^{\dagger} a_{\kappa-\nu}=\delta_{\kappa \lambda} \Pi_{\kappa},
\end{array}
$$

where $\Pi_{\kappa}$ is the projection on the subspace with particle number(s) $n$ determined by $\kappa$ through (3.14). We note that if $\nu$ is an irrational number, $n$ is uniquely determined by $\kappa$; if $\nu$ is a fraction $p / q, n$ is determined only modulo $q$ (assuming $p$ and $q$ do not have a common divisor). The projection $\Pi_{\kappa}$ satisfies the relations

$$
\begin{aligned}
& a_{\lambda}^{\dagger} \Pi_{\kappa}=\Pi_{\kappa+\nu} a_{\lambda}^{\dagger} \\
& a_{\lambda} \Pi_{\kappa}=\Pi_{\kappa-\nu} a_{\lambda}
\end{aligned}
$$

For rational $\nu=p / q$, we derive the following explicit expression for $\Pi_{\kappa}$,

$$
\Pi_{\kappa}=\int_{0}^{2 \pi} d \theta \sum_{l=0}^{\infty} e^{i q(\kappa-l-\nu N) \theta}=\frac{1}{q} \sum_{n=1}^{q} e^{2 \pi i n(\kappa-\nu N)},
$$

while for general $\nu$ there is no such simple formula.

The commutation relations (3.18) imply that for fermionic commutation relations for the original fields we have

$$
a_{\kappa+\nu}^{\dagger} a_{\kappa}^{\dagger}=0
$$


This leads, in this case, to the additional restriction $k_{i} \neq k_{j}$ for the quantum numbers $\kappa_{i}$ given in (3.16).

Since the field $\psi(\theta)$ satisfies the free field equation (3.7) and the operators $a_{\kappa}$ therefore have the simple time evolution

$$
a_{\kappa}(t)=e^{-i \kappa t} a_{\kappa},
$$

the states (3.15) are energy eigenstates with time evolution

$$
\left\langle\kappa_{1}, \ldots, \kappa_{N} \mid \Psi(t)\right\rangle=\frac{1}{\sqrt{N !}}\left\langle 0\left|a_{\kappa_{1}}(t) \ldots a_{\kappa_{N}}(t)\right| \Psi(0)\right\rangle=e^{-i \sum_{i} \kappa_{i} t}\left\langle\kappa_{1}, \ldots, \kappa_{N} \mid \Psi(0)\right\rangle .
$$

Thus, the energy (or angular momentum) eigenvalue of the state is simply

$$
E\left[\kappa_{1}, \ldots, \kappa_{N}\right]=\sum_{i} \kappa_{i}
$$

This permits an occupation number interpretation of the states (3.15) generated by the operators $a_{\kappa}^{\dagger}$. Such an operator creates a particle with energy $\kappa$, and the total energy is equal to the sum of single particle energies, just as if the particles were non-interacting. Note however that the $\nu$-dependent statistics interaction affects the values of the single particle energies, (3.16). The commutation relations (3.18) also show that the occupation number description is non-unique. A reordering of creation operators will give a different distribution over single particle levels, although the total energy remains invariant. This is illustrated in Fig.1 for two different but equivalent distributions in the case $\nu=1 / 3$. However, the distribution over single particle levels may be given a unique definition by introducing a normal ordering of creation operators. A possible definition of such a normal ordering of a string of creation operators $a_{\kappa_{N}}^{\dagger} \ldots a_{\kappa_{1}}^{\dagger}$, would be the requirement

$$
\begin{array}{ll}
\kappa_{i+1} \geq \kappa_{i}+\nu, & \text { Bose }, \\
\kappa_{i+1} \geq \kappa_{i}+\nu+1, & \text { Fermi } .
\end{array}
$$

This requirement can readily be seen to be satisfied for one of the possible orderings of the operators by use of the commutation relations (3.18).

To comment a bit further on this occupation number description, we will assume $\nu=p / q$, with $p$ and $q$ integers. If we compare with the energy levels of the $\nu=0$ system, we note that there is a splitting of $p$ of these levels into $q$ levels in the new system. This gives a typical level distance of $1 / q$ in the new system, although the level distance increases to $p / q$ at the bottom of the spectrum. However, these levels cannot be freely filled with particles. When filled from the bottom of the spectrum (according to normal ordering), there has to be a minimum distance of $p / q$ between one particle and the next, even if there are unoccupied levels in between. In terms of the levels of the $\nu=0$ system this is like having a maximum occupation of $q / p$ for each level, but the energies of these particles are not the same due to the level splitting between the $\nu=0$ and the $\nu=p / q$ systems. As an example, we show the ground state of the $\nu=4 / 5$ system in Fig.2. 
The occupation rules discussed above clearly suggest a connection to exclusion statistics, first introduced by Haldane [9]. This connection can be made explicit, as will be discussed in a separate publication.

\section{Field theory and the anyon droplet}

We will now consider a model Hamiltonian that incorporates several features of a real FQH system. The starting point is a system of "free" anyons in a strong magnetic field, $B$, and confined to a circular region by a harmonic potential $V(r)=e A_{0}=\frac{1}{2} m \omega^{2} r^{2}$. The potential is weak in the sense that $\omega \ll \omega_{c}$, where $\omega_{c}$ is the cyclotron frequency of the magnetic field. This theory is equivalent to having the particles moving in an effective magnetic field

$$
e B_{\mathrm{eff}}=\sqrt{(e B)^{2}+(2 m \omega)^{2}} \simeq e B+2 \frac{(m \omega)^{2}}{e B}
$$

with the Hamiltonian $H=a L+N \omega_{c}^{\text {eff }} / 2$ where $L$ is the total angular momentum operator, $\omega_{c}^{\text {eff }}$ the cyclotron frequency of the effective field, and

$$
a=\frac{1}{2 m}\left(e B_{\mathrm{eff}}-e B\right) \simeq \frac{m \omega^{2}}{e B}=\frac{\omega^{2}}{\omega_{c}} .
$$

We also have $a=v / R$ where $R$ is the radius of the anyon "droplet", and $v$ the "Fermi velocity", i.e. the drift velocity of the electrons at the edge due to the harmonic oscillator potential $e A_{0}$ (see (C.19)). The system is described by the following field theoretical Hamiltonian,

$$
H=a \int_{0}^{2 \pi} d \theta \chi^{\dagger}(\theta)\left[\left(-i \partial_{\theta}-\kappa_{F}\right)+\frac{\nu}{2} \int_{0}^{2 \pi} d \theta^{\prime} \chi^{\dagger}\left(\theta^{\prime}\right) \chi\left(\theta^{\prime}\right)\right] \chi(\theta)
$$

where the Fourier transform of $\chi(\theta)$ only includes positive frequency components. The Fermi (angular) momentum $\kappa_{F}$ has been introduced to fix the number of particles in the ground state. It corresponds to including a chemical potential

$$
\mu_{0}=a \kappa_{F}+\frac{1}{2} \omega_{c}^{\mathrm{eff}}
$$

Throughout this section, we choose to work in the fermionic representation. Then, the particle number $N_{0}$ of the ground state is determined by

$$
(\nu+1)\left(N_{0}-1\right) \leq \kappa_{F} \leq(\nu+1) N_{0}
$$

and for convenience we choose

$$
\kappa_{F}=(\nu+1)\left(N_{0}-\frac{1}{2}\right)
$$


The physical size of the droplet is

$$
R^{2} \approx r_{0}^{2}(\nu+1) N_{0} \approx r_{0}^{2} \kappa_{F}
$$

$r_{0}$ being the magnetic length $\sqrt{\frac{2}{e B_{e f f}}}$.

With the following transformation of the field

$$
\chi(\theta) \rightarrow \psi(\theta)=e^{i\left(\nu N-\kappa_{F}\right) \theta} \chi(\theta), \quad N=\int_{0}^{2 \pi} d \theta \psi^{\dagger}(\theta) \psi(\theta)
$$

the new field will satisfy the free field equation,

$$
\left(\partial_{t}+a \partial_{\theta}\right) \psi(\theta)=0
$$

and the total energy is simply a sum of single particle energies,

$$
E\left[\kappa_{1}, \ldots, \kappa_{N}\right]=a \sum_{i} \kappa_{i}
$$

In this case the possible values of $\kappa$ are determined by

$$
\kappa=k+\nu n-\kappa_{F}
$$

where $k$ and $n$ are nonnegative integers. The ground state corresponds to having the negative $\kappa$ states filled, in the way discussed in the previous section.

Expressed in terms of the $\psi$-field, we then have a free field theory with the form of a chiral, massless fermion theory on the circle. However, due to the non-trivial periodicity conditions, the angular momentum variable $\kappa$ takes unconventional values. There is also a "bottom" in the Fermi sea - it is defined by $\kappa=-\kappa_{F}$, and thus related to the total number of particles in the system. As pointed out in the previous section (see (3.12) ) the Hamiltonian does not have the free field form, but is given by

$$
H=a H_{L}=a \int_{0}^{2 \pi} d \theta \psi^{\dagger}(\theta)\left[-i \partial_{\theta}-\frac{\nu}{2} \int_{0}^{2 \pi} d \theta^{\prime} \psi^{\dagger}\left(\theta^{\prime}\right) \psi\left(\theta^{\prime}\right)\right] \psi(\theta) .
$$

The model discussed in this section has the form of a chiral Luttinger model, and for even values, $\nu=2 m$, it is essentially the same as the one used by Wen [6, 10 to describe the edge excitations of a droplet of fermions in the lowest Landau level (see also Stone [7]). In our case, however, the Hamiltonian gives an exact description of the full anyon system, and the excitations are in general not excitations of the edge. The "Fermi" surface $\kappa=0$ corresponds to the edge of the droplet, and small $\kappa$ will correspond to excitations close to this surface. But the field operator $\psi^{\dagger}$ also includes components (large positive $\kappa$ ) which create particles far from the surface, and components (large negative $\kappa$ ) which annihilate holes deep inside the droplet. One should also note that, due to the uncertainty relation between $\kappa$ and $\theta$, an excitation that is sharp in $\theta$ is totally spread out in angular momentum, or equivalently, totally delocalized in the radial coordinate $r$. 
We now demonstrate that if we ignore the finite size effects due to the "bottom" of the Fermi sea, and consider the special values $\nu=2 m$, our theory is exactly the one used by Wen to describe the edge of a quantum Hall system with filling fraction $1 /(2 m+1)$. We return to the formulation in terms of the original field $\chi$ and write the Hamiltonian (4.3) in a form which is normal ordered with respect to the Fermi level,

$$
H=a\left[\int_{0}^{2 \pi} d \theta: \chi^{\dagger}\left(-i \partial_{\theta}+\frac{1}{2}\right) \chi:+\frac{\nu}{2} Q^{2}\right]
$$

with $Q=N-N_{0}$ as the charge operator. Writing $H$ in this form we have redefined the field $\chi$ by absorbing a phase factor $e^{i N_{0} \theta}$ and subtracted the ground state energy. This theory can be bosonized using standard techniques (for all the relevant details see [11]). The bosonized form of the fermion operator is,

$$
\chi^{\dagger}(\theta, t)=\frac{e^{\frac{i}{2}(\theta-a t)}}{\sqrt{2 \pi}}: e^{i \phi(\theta, t)}:
$$

where the chiral Bose field $\phi$ can be written in the form,

$$
\phi(\theta, t)=\phi_{0}-Q(\theta-a t)+i \sum_{n>0} \frac{1}{\sqrt{n}}\left[a_{n} e^{i n(\theta-a t)}-a_{n}^{\dagger} e^{-i n(\theta-a t)}\right] \quad .
$$

The operator $\phi_{0}$ is conjugate to the charge operator $Q,\left[\phi_{0}, Q\right]=i$, and the normal ordering in (4.14) only refers to the operators $a_{n}$ and $a_{n}^{\dagger}$ that annihilate and create neutral chiral excitations and satisfy the commutation relation $\left[a_{m}, a_{n}^{\dagger}\right]=\delta_{m n}$. The charge $Q$ determines the periodicity of the Bose-field and can be interpreted as the winding number associated with this field. The Hamiltonian takes the bosonized form,

$$
\begin{aligned}
H & =a\left[\frac{1}{4 \pi} \int_{0}^{2 \pi} d \theta:\left(\partial_{\theta} \phi\right)^{2}:+m Q^{2}\right] \\
& =a\left[\sum_{n>0} n a_{n}^{\dagger} a_{n}+\frac{1}{2}(2 m+1) Q^{2}\right] .
\end{aligned}
$$

This Hamiltonian is precisely of the form discussed by Wen. However, the correlation functions of the fermion operators are obviously those of a free fermion theory and the short distance behaviour (which can also be interpreted as the large $R$ behavior) can be calculated using either the fermionic or bosonic form of $\chi$ and $\chi^{\dagger}$,

$$
\left\langle 0\left|\chi^{\dagger}(\theta, t) \chi(0, t)\right| 0\right\rangle=\frac{1}{2 \pi} \frac{1}{e^{-i \theta}-1} \sim \frac{i}{2 \pi} \frac{1}{\theta}
$$

Thus, the operator $\chi$ is not identical to the operator identified by Wen as the fermion operator [10]. To find the correspondence to Wen's fermion operator, we first note that we, by a change in the definition of the Bose field, can absorb the interaction term of the 
Hamiltonian and bring the full Hamiltonian to a free field form. The new Bose field is defined as

$$
\tilde{\phi}(\theta, t)=\frac{1}{\sqrt{2 m+1}} \phi_{0}-\sqrt{2 m+1} Q(\theta-a t)+i \sum_{n>0} \frac{1}{\sqrt{n}}\left[a_{n} e^{i n(\theta-a t)}-a_{n}^{\dagger} e^{-i n(\theta-a t)}\right]
$$

and the Hamiltonian has the free field form

$$
H=\frac{a}{4 \pi} \int_{0}^{2 \pi} d \theta:\left(\partial_{\theta} \tilde{\phi}\right)^{2}:
$$

The parameter $\nu=2 m$ now only appears implicitly in the periodicity conditions of the field $\tilde{\phi}$.

Corresponding to the Bose field $\tilde{\phi}$ we introduce the new fermion field

$$
\tilde{\chi}^{\dagger}(\theta, t)=\frac{e^{\frac{i}{2}(2 m+1)(\theta-a t)}}{\sqrt{2 \pi}}: e^{i \sqrt{(2 m+1)} \tilde{\phi}(\theta, t)}:
$$

This operator is the one which can be identified with Wen's fermion operator. The correlation functions for the operator do not correspond to free fermions (or a Fermi liquid) but are typically

$$
\left\langle 0\left|\tilde{\chi}^{\dagger}(\theta, t) \tilde{\chi}(0, t)\right| 0\right\rangle \sim(-1)^{m} \frac{i}{2 \pi} \frac{1}{\theta^{(2 m+1)}}
$$

However, it may be important to note that this behavior is closely related to the fact that the anticommutator $\left\{\tilde{\chi}^{\dagger}(\theta, t), \tilde{\chi}\left(\theta^{\prime}, t\right)\right\}$ does not equal a (periodic) delta function, but a more complicated local distribution involving derivatives of the delta function. In this respect it is not a standard fermion operator. We also note that the Hamiltonian (4.19) does not have simple form when expresssed in terms of $\tilde{\chi}$ and $\tilde{\chi}^{\dagger}$. Thus, the form of the correlation function (4.21) is not sufficient to imply a strongly correlated ground state of Luttinger liquid type in the present model. This is not to say that the real electrons at a FQH edge do not form a Luttinger liquid. Remember that the fields in our simple model on the circle are related to the original anyons in the lowest Landau level by the transformation $S$ (see (2.27)-(2.30) ) which is not explicitly known. It is not inconceivable that the correlation functions of this anyon operator are more closely related to the correlation functions of the operator $\tilde{\chi}$ rather than those of the field $\chi$, which describes the "elementary" fermions in our simple model. Clearly this is an interesting and important point to clarify. (For a related discussion see [12].)

As stressed by Wen, the thermodynamic properties of the edge states in the QH system are the same as for a Fermi liquid and we expect the same in our model. In Appendix C we derive an exact formula for the grand canonical partition function for our model on the circle and show that the specific heat at low $T$ is the same as the one derived by Wen for the QH system. 
Finally, we point out that the transformed field $\psi$ (4.8), which satisfies the free field equation and obeys the modified commutators (3.8), can also be related to the Bose field $\phi$ in a simple way. For arbitrary $\nu$ we have

$$
\psi^{\dagger}(\theta, t)=\frac{1}{\sqrt{2 \pi}}: e^{i(\phi-\nu Q(\theta-a t))}:
$$

and the bosonized form of the Hamiltonian is as given by (4.16) with $2 m$ replaced by $\nu$.

\section{Concluding remarks}

In this paper we have exploited the one-dimensional nature of the lowest Landau level to formulate a field theory of anyons. The natural Hamiltonian in this theory is the total angular momentum, which is essentially the energy operator in a harmonic oscillator well. The field theory is expressed with the holomorphic field operator $\varphi(z)$ and the antiholomorphic $\varphi^{\dagger}(\bar{z})$ as the fundamental variables. In terms of these operators the angular momentum takes a simple form.

We have also formulated the theory as a function of a real variable on a circle. In this formulation we cast the theory in free form by a non-local transformation on the fields. However, the non-locality is of a rather simple nature since it only enters via the total particle number $N$, and the transformed fields can be shown to satisfy rather simple, modified commutation relations. The corresponding Fock space structure is interesting in that it allows for an occupation number interpretation of the energy eigenstates. For a given state this interpretation is not unique, but must be specified by an ordering convention for the single particle states.

The $N$-dependent interaction can be interpreted as a kind of "statistical" interaction between particles with fractional statistics on the circle. There exists another realization of fractional statistics in one dimension that we have so far not mentioned, namely the one related to the Calogero model [13, 14]. This is a system of $N$ particles in one dimension interacting by a repulsive ("statistical") $1 / x^{2}$ two-body potential. Also this model has been shown to be equivalent to the system of anyons in the lowest Landau level [15, 16], although again with no simple transformation between the two systems [17, 18. The correspondences to the anyons in the lowest Landau level suggest a close connection between the two different realizations of fractional statistics in one dimension. However, we have not found any explicit connection between the field theory considered in this paper and the second quantized version of the Calogero model.

The theory discussed in this paper describes a system of non-interacting anyons in a strong magnetic field. It is related to the system of interacting particles in the sense that anyons are supposed to correspond to elementary excitations in an incompressible Hall fluid. As pointed out, the theory is identical to Wen's theory of the edge excitations in the simplest FQH fluids. An interesting question is whether corrections can be made by introducing particle interactions explicitly in the description. In particular, can the onedimensionality of the system be exploited to simplify such a description? This is not so

obvious, since the interactions, when projected into the lowest Landau level, may take a 
rather complicated form. This is seen already in the case of interacting fermions, where two-body interaction potentials in the full theory are mapped into functions of derivatives in the projected theory [19]. In the general anyon case this projection takes an even more complicated form. Thus, the gain obtained by reduction of dimension will in general be compensated by a more complicated form of the interaction Hamiltonian and of other observables.

However, some special types of interaction may appear in a simple form in the onedimensional theory. The full anyon system is periodic in the statistics parameter $\nu$, so in the one-dimensional description, $\nu$ is effectively constrained to $0 \leq \nu<2$. The extension to larger values of $\nu$, discussed at the end of section 4 in the context of the edge theory, should be interpreted as describing an anyon system with a special short range repulsion which excludes certain states from the spectrum. In particular, the odd-integer values of $\nu$ larger than 1 correspond to fermions with a short range repulsion of the form discussed by Haldane [20] and by Trugman and Kivelson [21]. This additional repulsion can be viewed as due to a magnetic flux attatched to each particle, which corresponds to the "composite fermion" picture of Jain [22]. We may hope that the free-field form of the anyon theory discussed here may be useful to examine further certain aspects of the many-particle system with a repulsive interaction between the particles, but as already stressed, the real challenge is to derive the one-dimensional expressions for the original lowest Landau level electron operators (and for a general $\nu$ for the original anyon operators).

\section{Acknowledgments}

We would like to thank S. Isakov for useful discussions and comments.

\section{Appendix A: From full field theory to the lowest Landau level}

We shall connect the field theory defined in Section 2 to the full field theory of anyons in a magnetic field. This problem has been studied earlier in the case of fermions in e.g. [23]. For the full theory of anyons in a magnetic field a (non-local) field theory can be defined in terms of bosonic operators $\Phi(z, \bar{z})$ with a statistics interaction included in the Hamiltonian,

$$
H=-\frac{e B}{2 m} \int d^{2} z \Phi^{\dagger}(z, \bar{z})[D \bar{D}+\bar{D} D] \Phi(z, \bar{z})
$$

Here $D$ and $\bar{D}$ are covariant derivatives, $D=\partial-i A$ and $\bar{D}=\bar{\partial}-i \bar{A}$, with

$$
A=A_{\text {stat }}+A_{\text {ext }}=i \frac{\nu}{2} \int d^{2} z^{\prime} \rho\left(z^{\prime}, \bar{z}^{\prime}\right) \frac{1}{z-z^{\prime}}-i \frac{\bar{z}}{2}
$$

where $\rho(z, \bar{z})=\Phi^{\dagger}(z, \bar{z}) \Phi(z, \bar{z})$ is the density operator. Similarly, for the total angular momentum we have

$$
L=\int d^{2} z \Phi^{\dagger}(z, \bar{z})[z D-\bar{z} \bar{D}+\bar{z} z] \Phi(z, \bar{z}) .
$$


A wave function $\Psi$ in the lowest Landau level obeys the condition

$$
\bar{D}_{i} \Psi(z, \bar{z})=\left(\bar{\partial}_{i}+\frac{z_{i}}{2}+\frac{\nu}{2} \sum_{j \neq i} \frac{1}{\bar{z}_{j}-\bar{z}_{i}}\right) \Psi(z, \bar{z})=0
$$

This can be translated into the following condition which involves the field operator,

$$
\bar{D} \Phi(z, \bar{z}) P=0
$$

where $P$ denotes the projection on the lowest Landau level. (This can in fact be regarded as the defining equation for the projection $P$ in the field theoretical formulation.) For the projection of the Hamiltonian we get

$$
\begin{aligned}
P H P & =-\frac{e B}{m} \int d^{2} z P \Phi^{\dagger}(z, \bar{z})\left[D \bar{D}-\frac{1}{2}\right] \Phi(z, \bar{z}) P \\
& =\frac{e B}{2 m} \int d^{2} z P \Phi^{\dagger}(z, \bar{z}) \Phi(z, \bar{z}) P .
\end{aligned}
$$

When deriving (A.6) from (A.1) one seems to pick up an additional contact term $\sim \int d^{2} z P \Phi^{\dagger} \Phi^{\dagger} \Phi \Phi P$ from the commutator $[D, \bar{D}]$, but this term vanishes due to the projection on the lowest Landau level. This follows from the fact that, for states in the lowest Landau level, the operator $\Phi\left(z^{\prime}, \bar{z}^{\prime}\right) \Phi(z, \bar{z}) P$ vanishes like $\left|z-z^{\prime}\right|^{\nu}$ when $z^{\prime}$ approaches $z$. This is shown explicitly in (A.9). .

The expression (A.6) is, except for the prefactor $e B / 2 m$, identical to the number operator for anyons in the lowest Landau level. In terms of the field operators introduced in Section 2, the projected Hamiltonian then gets the form

$$
P H P=\frac{e B}{2 m} \int d^{2} z e^{-\bar{z} z} \phi^{+}(\bar{z}) \phi(z)=\frac{e B}{2 m} \int d^{2} z e^{-\bar{z} z} \varphi^{\dagger}(\bar{z}) \varphi(z) .
$$

The projection of the angular momentum is

$$
\begin{aligned}
P L P= & \int d^{2} z P \Phi^{\dagger}(z, \bar{z})[z D-\bar{z} \bar{D}+\bar{z} z] \Phi(z, \bar{z}) P \\
= & \int d^{2} z P \Phi^{\dagger}(z, \bar{z}) z\left(\partial+\frac{\bar{z}}{2}\right) \Phi(z, \bar{z}) P \\
& +\frac{\nu}{4} \int d^{2} z \int d^{2} z^{\prime} P \Phi^{\dagger}(z, \bar{z}) \Phi^{\dagger}\left(z^{\prime}, \bar{z}^{\prime}\right) \Phi\left(z^{\prime}, \bar{z}^{\prime}\right) \Phi(z, \bar{z}) P .
\end{aligned}
$$

There is a clear resemblance between this expression for the projected operator $P L P$ and the corresponding expression (2.26) for the angular momentum, which involves the field operators $\phi(z)$ or $\phi^{+}(z)$, and which is derived directly from the $N$-particle description in the lowest Landau level. (Note however the factor $\nu / 4$ in the expression for PLP.) To

\footnotetext{
${ }^{1}$ For the full anyon theory the possible presence of an additional contact interaction, and its connection to the singular statistics interaction, is a somewhat subtle point. For discussions of this point see [24].
} 
examine further the relation between these expressions, we consider the connection between the projected field operators and the field operators in the lowest Landau level. We have

$$
\left\langle 0\left|\Phi\left(z_{1}, \bar{z}_{1}\right) \ldots \Phi\left(z_{N}, \bar{z}_{N}\right)\right| \psi\right\rangle=e^{-\frac{1}{2} \sum_{i} \bar{z}_{i} z_{i}} \prod_{i<j}\left|z_{i}-z_{j}\right|^{\nu}\left\langle 0\left|\phi\left(z_{1}\right) \ldots \phi\left(z_{N}\right)\right| \psi\right\rangle
$$

for an arbitrary state $|\psi\rangle$ in the lowest Landau level. This can be expressed as

$$
P \Phi^{\dagger}\left(z_{1}, \bar{z}_{1}\right) \ldots \Phi^{\dagger}\left(z_{N}, \bar{z}_{N}\right)|0\rangle=e^{-\frac{1}{2} \sum_{i} \bar{z}_{i} z_{i}} \prod_{i<j}\left|z_{i}-z_{j}\right|^{\nu} \phi^{\dagger}\left(\bar{z}_{1}\right) \ldots \phi^{\dagger}\left(\bar{z}_{N}\right)|0\rangle .
$$

¿From this we derive

$$
\begin{aligned}
P \Phi^{\dagger}\left(z_{1}, \bar{z}_{1}\right) & \ldots \Phi^{\dagger}\left(z_{N}, \bar{z}_{N}\right)|0\rangle=e^{-\frac{1}{2} \bar{z}_{1} z_{1}} \prod_{i \neq 1}\left|z_{1}-z_{i}\right|^{\nu} \phi^{\dagger}\left(\bar{z}_{1}\right) P \Phi^{\dagger}\left(z_{2}, \bar{z}_{2}\right) \ldots \Phi^{\dagger}\left(z_{N}, \bar{z}_{N}\right)|0\rangle \\
& =e^{-\frac{1}{2} \bar{z}_{1} z_{1}} \phi^{\dagger}\left(\bar{z}_{1}\right) P e^{\nu \int d^{2} z \ln \left|z_{1}-z\right| \Phi^{\dagger}(z, \bar{z}) \Phi(z, \bar{z})} \Phi^{\dagger}\left(z_{2}, \bar{z}_{2}\right) \ldots \Phi^{\dagger}\left(z_{N}, \bar{z}_{N}\right)|0\rangle(\mathrm{A} .11)
\end{aligned}
$$

which implies

$$
P \Phi^{\dagger}(z, \bar{z})=e^{-\frac{1}{2} \bar{z} z} \phi^{\dagger}(\bar{z}) P e^{\nu \int d^{2} z^{\prime} \ln \left|z-z^{\prime}\right| \Phi^{\dagger}\left(z^{\prime}, \bar{z}^{\prime}\right) \Phi\left(z^{\prime}, \bar{z}^{\prime}\right)} .
$$

We introduce the operators

$$
\Sigma(z, \bar{z})=\nu \int d^{2} z^{\prime} \ln \left|z-z^{\prime}\right| \Phi^{\dagger}\left(z^{\prime}, \bar{z}^{\prime}\right) \Phi\left(z^{\prime}, \bar{z}^{\prime}\right)
$$

and

$$
\begin{aligned}
\Delta & =\int d^{2} z \Phi^{\dagger}(z, \bar{z}) \Sigma(z, \bar{z}) \Phi(z, \bar{z}) \\
& =\nu \int d^{2} z \int d^{2} z^{\prime} \ln \left|z-z^{\prime}\right| \Phi^{\dagger}(z, \bar{z}) \Phi^{\dagger}\left(z^{\prime}, \bar{z}^{\prime}\right) \Phi\left(z^{\prime}, \bar{z}^{\prime}\right) \Phi(z, \bar{z})
\end{aligned}
$$

and the transformed field

$$
\begin{aligned}
\tilde{\Phi}(z, \bar{z}) & =e^{\left[\frac{1}{2} \bar{z} z-\Sigma(z, \bar{z})\right]} \Phi(z, \bar{z}) \\
& =e^{\frac{1}{2} \bar{z} z} e^{\frac{\Delta}{2}} \Phi(z, \bar{z}) e^{-\frac{\Delta}{2}}
\end{aligned}
$$

The operator $\tilde{\Phi}$ satisfies the condition

$$
\bar{\partial} \tilde{\Phi}(z, \bar{z}) P=0
$$

as can be verified directly from the condition (A.5). As follows from (A.12) the field operator $\phi(z)$ is simply the projection of $\tilde{\Phi}$ on the lowest Landau level,

$$
\phi(z)=\tilde{\Phi}(z, \bar{z}) P=P \tilde{\Phi}(z, \bar{z}) P .
$$


A corresponding expression can also be found for the dual field operator $\phi^{+}$. We then take (2.20) as our starting point and manipulate it in a similar way as done above to find the operator $\phi$. We get

$$
\begin{aligned}
\phi^{+}(\bar{z}) & =\frac{1}{\pi} \int d^{2} z^{\prime} e^{\left(\bar{z}-\frac{1}{2} \bar{z}^{\prime}\right) z^{\prime}} P \Phi^{\dagger}\left(z^{\prime}, \bar{z}^{\prime}\right) e^{\Sigma\left(z^{\prime}, \bar{z}^{\prime}\right)} P \\
& =\frac{1}{\pi} \int d^{2} z^{\prime} e^{\left(\bar{z}-\bar{z}^{\prime}\right) z^{\prime}} \phi^{\dagger}\left(\bar{z}^{\prime}\right) P e^{2 \Sigma\left(z^{\prime}, \bar{z}^{\prime}\right)} P .
\end{aligned}
$$

In the first of these expressions for $\phi^{+}$, the projection $P$ to the left can in fact be omitted when $P$ is present to the right. This is verified by showing that $\bar{D} \Phi(z, \bar{z})$ gives zero when applied to the expression. According to (A.5), this implies that the operator leaves the lowest Landau level invariant, and an explicit projection is not needed. By use of this property, and the corresponding one (A.17) for $\phi$, we can verify that the operators $\phi$ and $\phi^{+}$indeed satisfy the commutation relation (2.22). We can furthermore show that the expressions (A.6) and (A.8) for the projected Hamiltonian and angular momentum reproduce the correct form of $H$ and $L$, when these are expressed in terms of the operators $\phi(z)$ and $\phi^{+}(\bar{z})$ (see (2.25) and (2.26)).

It is of interest to note that the projection operators in fact can be left out altogether, when we consider the action of the operators on the subspace generated from the vacuum state by the action of $\phi^{+}$. We then write the field operators in the following way,

$$
\begin{aligned}
\phi(z) & =\frac{1}{\pi} \int d^{2} z^{\prime} e^{\left(z-\frac{1}{2} z^{\prime}\right) \bar{z}^{\prime}} e^{-\Sigma\left(z^{\prime}, \bar{z}^{\prime}\right)} \Phi\left(z^{\prime}, \bar{z}^{\prime}\right) \\
\phi^{+}(\bar{z}) & =\frac{1}{\pi} \int d^{2} z^{\prime} e^{\left(\bar{z}-\frac{1}{2} \bar{z}^{\prime}\right) z^{\prime}} \Phi^{\dagger}\left(z^{\prime}, \bar{z}^{\prime}\right) e^{\Sigma\left(z^{\prime}, \bar{z}^{\prime}\right)} .
\end{aligned}
$$

This is so since the vacuum state belongs to the subspace projected out by $P$, and this subspace is invariant under the action of $\phi$ and $\phi^{+}$, as explained above.

\section{Appendix B: Lagrangian formulation}

In this appendix we consider the classical mechanics of the Hamiltonian $H_{L}$ given in (3.4). In particular we shall derive the Poisson brackets and compare them to the commutators (3.8) derived in the text. The Lagrangian corresponding to $H_{L}$ is

$$
L_{L}=i \int_{0}^{2 \pi} d \theta \bar{\chi}(\theta) \dot{\chi}(\theta)-H_{L}(\chi, \bar{\chi})
$$

and by direct variation we obtain the equation of motion

$$
\left(i \partial_{t}+i \partial_{\theta}-\nu N\right) \chi(\theta)=0
$$

By the transformation

$$
\psi(\theta)=e^{i \nu N \theta} \chi(\theta) \quad \bar{\psi}(\theta)=e^{-i \nu N \theta} \bar{\chi}(\theta)
$$


where $N=\int_{0}^{2 \pi} d \theta \bar{\chi}(\theta) \chi(\theta)$, (B.2) becomes a free field equation for $\psi$.

The Poisson brackets between $\chi$ and $\bar{\chi}$ are canonical,

$$
\{\chi(\alpha), \bar{\chi}(\beta)\}=-i \delta_{p e r}^{+}(\alpha-\beta) \quad,
$$

and we calculate the Poisson brackets between $\psi$ and $\bar{\psi}$ as

$$
\begin{aligned}
\left\{\psi(\theta), \bar{\psi}\left(\theta^{\prime}\right)\right\} & =\int_{0}^{2 \pi} d \alpha d \beta\left[\{\chi(\alpha), \bar{\chi}(\beta)\} \frac{\delta \psi(\theta)}{\delta \chi(\alpha)} \frac{\delta \bar{\psi}\left(\theta^{\prime}\right)}{\delta \bar{\chi}(\beta)}+\{\bar{\chi}(\alpha), \chi(\beta)\} \frac{\delta \psi(\theta)}{\delta \bar{\chi}(\alpha)} \frac{\delta \bar{\psi}\left(\theta^{\prime}\right)}{\delta \chi(\beta)}\right] \\
& =-i \Delta\left(\theta-\theta^{\prime}\right)+\nu\left(\theta-\theta^{\prime}\right) \bar{\psi}\left(\theta^{\prime}\right) \psi(\theta)
\end{aligned}
$$

Using a similar expression we also get

$$
\left\{\psi(\theta), \psi\left(\theta^{\prime}\right)\right\}=-\nu\left(\theta-\theta^{\prime}\right) \psi\left(\theta^{\prime}\right) \psi(\theta) \quad .
$$

In deriving these expressions, it is important to keep the correct sign of the argument in the positive frequency delta function $\delta_{p e r}^{+}(\alpha-\beta)$ and to use relations like (3.10) and (3.11) to perform the integrals.

If we quantize by the canonical prescription $i\{A, B\} \rightarrow[A, B]$, we get

$$
\begin{aligned}
\psi(\theta) \psi\left(\theta^{\prime}\right) & -\left[1-i \nu\left(\theta-\theta^{\prime}\right)\right] \psi\left(\theta^{\prime}\right) \psi(\theta)=0 \\
\psi^{\dagger}(\theta) \psi^{\dagger}\left(\theta^{\prime}\right) & -\left[1-i \nu\left(\theta-\theta^{\prime}\right)\right] \psi^{\dagger}\left(\theta^{\prime}\right) \psi^{\dagger}(\theta)=0 \\
\psi(\theta) \psi^{\dagger}\left(\theta^{\prime}\right) & -\left[1+i \nu\left(\theta-\theta^{\prime}\right)\right] \psi^{\dagger}\left(\theta^{\prime}\right) \psi(\theta)=\Delta\left(\theta-\theta^{\prime}\right)
\end{aligned}
$$

which agrees with the Bose part of the commutation relations (3.8) to leading order in $\nu$. Also notice that to $\mathcal{O}(\nu)$ there is no ordering ambiguity in $(\mathbb{B} .7)$. It thus seems that the classical limit must be defined by taking both $\hbar$ and $\nu$ to zero, and then, in a sense, $H_{L}$ together with (B.7) describes "classical anyons" for small $\nu$.

\section{Appendix C: Statistical Mechanics}

In this appendix we derive the grand canonical partition function of the system of particles on the circle discussed in Section 4. Again, we choose $\nu=0$ to correspond to fermions.

The general expression for the grand canonical partition function is

$$
\Xi[\beta, \mu, V]=\sum_{N=1}^{\infty} \sum_{\left\{n_{k}\right\}} \exp [-\beta(E-\mu N)],
$$

where $\left\{n_{k}\right\}$ denotes all possible configurations of the $N$-particle system. We know from Section 4 that the total energy of $N$ anyons in our system has the form

$$
E=a\left(\sum_{k=0}^{\infty} k n_{k}+\frac{\nu}{2} N(N-1)\right)+\frac{N}{2} \omega_{c}^{e f f} .
$$


(Here the ground state energy has not been subtracted.) Since we are working in the fermionic representation, the occupation numbers $n_{k}$ can take the values 0 and 1 .

Now we move the zero-point of the energy up to the Fermi level, which is assumed to be large. In this way we obtain a more symmetric picture with a deep Fermi sea and both particle- and hole states. As in Section 4, we rewrite $N \equiv Q+N_{0}$, where $N_{0}$ is some large number, and

$$
\begin{aligned}
\sum_{k=0}^{\infty} k n_{k} & =\sum_{k=0}^{N_{0}-1} k n_{k}+\sum_{k=N_{0}}^{\infty} k n_{k} \\
& =\sum_{l=0}^{\infty}\left(l+N_{0}\right) n_{l}^{+}+\sum_{l=0}^{N_{0}-1}\left(l-N_{0}+1\right) n_{l}^{-}+\frac{1}{2} N_{0}\left(N_{0}-1\right)
\end{aligned}
$$

where we have identified the particle- and hole occupation numbers as $n_{l}^{+} \equiv n_{N_{0}+l}$ and $n_{l}^{-} \equiv 1-n_{N_{0}-1-l}$ respectively. The charge, i.e. the total number of particles with $N_{0}$ subtracted, is now given by $Q=\sum_{l=0}^{\infty} n_{l}^{+}-\sum_{l=0}^{N_{0}-1} n_{l}^{-}$. Similarly, we rewrite the statistical interaction term,

$$
\frac{1}{2} \nu N(N-1)=\frac{1}{2} \nu Q^{2}+\frac{1}{2} \nu Q\left(2 N_{0}-1\right)+\frac{1}{2} \nu N_{0}\left(N_{0}-1\right)
$$

and $\mu N=\mu Q+\mu N_{0}$. This gives

$$
\begin{aligned}
E-\mu N & =\sum_{l=0}^{N_{0}-1}\left[a\left(l+\frac{1}{2}\right)+\mu_{e f f}\right] n_{l}^{-}+\sum_{l=0}^{\infty}\left[a\left(l+\frac{1}{2}\right)-\mu_{e f f}\right] n_{l}^{+} \\
& +\frac{1}{2} a \nu Q^{2}+\Omega_{0}
\end{aligned}
$$

where the effective chemical potential is given by

$$
\begin{aligned}
\mu_{e f f} & =\mu-a(\nu+1)\left(N_{0}-\frac{1}{2}\right)-\frac{1}{2} \omega_{c}^{\text {eff }} \\
& =\mu-\mu_{0}
\end{aligned}
$$

and

$$
\Omega_{0}=a \frac{1}{2}(\nu+1) N_{0}\left(N_{0}-1\right)-\left(\mu-\frac{1}{2} \omega_{c}^{e f f}\right) N_{0} .
$$

Now, assume that $N_{0}$ is so large that it can be replaced by infinity in the sums above. Then, except for the $Q^{2}$ term, our model looks like a fermionic system of particles and holes with odd-integer energy levels (with spacing $a / 2$ ), an effective chemical potential $\mu_{\text {eff }}$ and a zero-point energy $\Omega_{0}$. The troublesome term $\exp \left(-\beta \nu a Q^{2} / 2\right)$ in the partition function can be dealt with by rewriting it as a Gaussian integral (Hubbard-Stratonovich transformation),

$$
\exp \left[-\frac{1}{2} \beta \nu a Q^{2}\right]=\sqrt{\frac{\beta \nu a}{2 \pi}} \int_{-\infty}^{\infty} d \sigma \exp \left[-\frac{1}{2} \beta \nu a \sigma^{2}-i \beta \nu a Q \sigma\right],
$$


leaving us with a term which is only linear in $Q$, thus giving an imaginary contribution to the effective chemical potential which becomes $\mu_{e f f}-i \nu a \sigma$. In summary, our partition function is now given by

$\Xi^{\nu}=\exp \left[-\beta \Omega_{0}\right] \sqrt{\frac{\beta \nu a}{2 \pi}} \int_{-\infty}^{\infty} d \sigma \exp \left[-\frac{1}{2} \beta \nu a \sigma^{2}\right] \cdot \Xi_{F}\left[\beta, \mu_{e f f}-i \nu a \sigma\right] \cdot \Xi_{F}\left[\beta,-\left(\mu_{e f f}-i \nu a \sigma\right)\right]$,

where the fermionic partition functions are of the usual form

$$
\Xi_{F}[\beta, \mu]=\prod_{l}\left(1+e^{-\beta\left(\epsilon_{l}-\mu\right)}\right)
$$

where, in our case, $\epsilon_{l}=a(2 l+1) / 2$. This expression can be rewritten in terms of the function $\Theta_{3}(u, q)$, defined by 25]

$$
\begin{aligned}
\Theta_{3}(u, q) & =\prod_{n=0}^{\infty}\left(1+e^{2 i u} q^{2 n+1}\right)\left(1+e^{-2 i u} q^{2 n+1}\right) \prod_{n=1}^{\infty}\left(1-q^{2 n}\right) \\
& =\sum_{n=-\infty}^{\infty} q^{n^{2}} e^{2 i n u}
\end{aligned}
$$

where we identify $q=\exp [-\beta a / 2]$ and $u=\frac{1}{2}\left(\beta \nu a \sigma-i \beta \mu_{e f f}\right)$. We use the series representation of the $\Theta$ function to perform the integral in (C.12) term by term, identifying the last product of $\Theta_{3}$ as the inverse of a bosonic partition function with integer energy levels (in units of $a$ ) and zero chemical potential. The series obtained after integrating over $\sigma$ is again a $\Theta$ function and can thus be written as a product of partition functions as discussed above. The result of this calculation is

$$
\Xi^{\nu}=e^{-\beta \Omega_{0}} \Xi_{F}^{\nu+1}\left[\beta, \mu_{e f f}\right] \cdot \Xi_{F}^{\nu+1}\left[\beta,-\mu_{e f f}\right]\left(\Xi_{B}^{\nu+1}[\beta, \mu=0]\right)^{-1} \Xi_{B}[\beta, \mu=0],
$$

where

$$
\begin{aligned}
\Xi_{F}^{1+\nu}\left[\beta, \mu_{e f f}\right] & =\prod_{n=0}^{\infty}\left(1+\exp -\beta\left[\frac{2 n+1}{2} a(\nu+1)-\mu_{e f f}\right]\right) \\
\Xi_{B}^{1+\nu}[\beta, \mu=0] & =\prod_{n=1}^{\infty}(1-\exp -\beta[\operatorname{an}(1+\nu)])^{-1} .
\end{aligned}
$$

Thus, we have obtained an expression for the partition function of the system of particles on the circle simply as a product of fermionic and bosonic partition functions with shifted chemical potential and rescaled energy levels and a prefactor determined by the offset $\Omega_{0}$. Taking the fermionic limit $\nu=0$ in (C.16), we see that the two bosonic partition functions cancel and we are, apart from the offset, left with a product of the fermionic particle- and hole partition functions (characterized by $\mu>0$ and $\mu<0$ respectively). 
A direct application of this result is the computation of the specific heat in the low temperature limit. To specify how to take the thermodynamic limit of the system, we write the parameter $a$ which defines the energy splitting in the following form,

$$
a \equiv \frac{2 \pi}{L} v \approx \frac{\omega^{2}}{\omega_{c}}
$$

The length $L$, which we interpret as the circumference of the circle in the one-dimensional description, is defined as the length of the edge of the anyon droplet in the harmonic oscillator potential, $L=2 \pi R$. $v$ then corresponds to the drift velocity of the charged particles at the edge. The large "volume" limit, $L \rightarrow \infty$ is taken such that $v$ stays finite. This implies that both $\omega$ and $a$ go to zero in the thermodynamic limit.

Our strategy now is to expand the thermodynamic potential, which is $-k T$ times the logarithm of the partition function, in powers of the temperature,

$$
\Omega=\Omega_{0}+c_{1}(\mu, L) T+c_{2}(\mu, L) T^{2}+\cdots
$$

and to combine the following expressions for the entropy and the specific heat,

$$
\begin{aligned}
S & =-\left(\frac{\partial \Omega}{\partial T}\right)_{V, \mu} \\
C_{V} & =T\left(\frac{\partial S}{\partial T}\right)_{V, N} .
\end{aligned}
$$

In calculating the thermodynamic potential corresponding to (C.16), we get two bosonic and two fermionic contributions, plus a $T$-independent term $\Omega_{0}$. The sums over energy levels can be approximated by integrals, choosing as the integration variable $x=\beta$ an, such that $d x=\beta a d n=\beta a$, which is small at a given $T$ in the thermodynamic limit $\omega \rightarrow 0$ $(a \rightarrow 0)$. Then, the two bosonic contributions become

$$
\begin{aligned}
-k T \ln Z_{B}[\beta] & =k T \sum_{n=0}^{\infty} \ln \left(1-e^{-\beta a n}\right) \\
& \approx \frac{(k T)^{2}}{a} \int_{0}^{\infty} d x \ln \left(1-e^{-x}\right)=-\frac{\pi^{2}}{6} \frac{(k T)^{2}}{a}
\end{aligned}
$$

and similarly,

$$
-k T \ln \left(Z_{B}^{\nu+1}\right)^{-1}=\frac{\pi^{2}}{6} \frac{(k T)^{2}}{a(\nu+1)}
$$

In a similar manner, we approximate the fermionic potential by

$$
-k T \ln Z_{F}^{\nu+1}\left[\beta, \mu_{e f f}\right] \approx-\frac{(k T)^{2}}{a(\nu+1)} \int_{0}^{\infty} d x \ln \left(1+\alpha e^{-x}\right), \quad \alpha=e^{\beta \mu_{e f f}} .
$$


If $\mu_{\text {eff }}<0$ then $\alpha \rightarrow 0$ in the low- $T$ limit, and the expression (C.26) becomes exponentially small. This implies that the contribution from the partition function of the particles vanishes for $T \rightarrow 0$, but for the partition function of the holes, which has exactly the same form, except for the sign of the chemical potential, there will be a non-vanishing contribution. For $\mu_{e f f}>0$ the situation is reversed, with a contribution only from the particles. There is an obvious symmetry of the total expression when the sign of $\mu_{e f f}$ is changed. Thus, let us assume that the effective chemical potential is positive such that the hole contribution is suppressed. Then, the integral in (C.26) can be calculated by rewriting it in the following way:

$$
\begin{aligned}
\int_{0}^{\infty} d x \ln \left(1+\alpha e^{-x}\right) & =\int_{0}^{\alpha} \frac{d y}{y} \ln (1+y) \\
& =\int_{0}^{1} \frac{d y}{y} \ln (1+y)+\int_{1}^{\alpha} \frac{d y}{y} \ln y+\int_{1}^{\alpha} \frac{d y}{y} \ln \left(1+\frac{1}{y}\right) \\
& =\frac{\pi^{2}}{12}+\frac{1}{2}\left(\beta \mu_{e f f}\right)^{2}+\frac{\pi^{2}}{12}+\mathcal{O}\left(e^{-\beta \mu_{e f f}}\right) .
\end{aligned}
$$

Thus, we obtain the following expression for the thermodynamic potential of the total partition function:

$$
\Omega^{\nu}=\Omega_{0}+\frac{\mu_{e f f}^{2}}{2 a(\nu+1)}-\frac{\pi^{2}}{6} \frac{(k T)^{2}}{a}+\cdots .
$$

Note that there is no first order term in $T$. (C.21) and (C.22) then imply that, to lowest order, $C_{V}$ is equal to the entropy. Also note that there is no statistics dependence in this approximation. To lowest order in $T$ the chemical potential $\mu_{e f f}$ is, for fixed density, independent of $T$. Differentiating (C.28) with respect to $T$ and making the substitution

$$
a \rightarrow \frac{2 \pi}{L} v
$$

we then find get the following expression for the heat capacity per unit length,

$$
c_{V}=\frac{C_{V}}{L}=\frac{\pi}{6} k^{2} \frac{T}{v} .
$$

This is identical to Wen's result for the specific heat of edge excitations in QH states, found from a hydrodynamical approach [6]. 


\section{References}

[1] R.E. Prange and S.M. Girvin, eds., The Quantum Hall Effect, Springer Verlag, 1987.

[2] G.W. Semenoff, Phys. Rev. Lett. 61, 517 (1988).

[3] G.W. Semenoff and P. Sodano, Nucl. Phys. B328, 753 (1989).

[4] T. Matsuyama, Phys. Lett. B228, 99 (1989).

[5] R. Banerjee, A. Chatterjee and V.V. Sreedhar, Ann. Phys. 222, 254 (1993).

[6] For a rewiev, see X.-G. Wen, Int. Jour. Mod. Phys B6, 1711(1992).

[7] M. Stone, Ann. Phys. 207, 38 (1991).

[8] E. Fradkin, Field Theories of Condensed Matter Systems, Addison-Wesley, 1991.

[9] F.D.M. Haldane, Phys. Rev. Lett. 67, 937 (1991).

[10] X.-G. Wen, Phys. Rev. B41, 12838 (1990).

[11] F.D.M. Haldane, J.Phys. C14, 2585 (1981).

[12] M. Stone and M.P.A. Fisher, preprint NSF-ITP-94-15, cond-mat 9402040

[13] J.M. Leinaas and J. Myrheim, Phys. Rev. B37, 9286 (1988).

[14] A.P. Polychronakos, Nucl. Phys. B324, 597 (1989).

[15] T.H. Hansson, J.M. Leinaas and J. Myrheim, Nucl. Phys. B384, 559 (1992).

[16] L. Brink, T.H. Hansson, S. Konstein and M. A. Vasiliev Nucl. Phys. B401, 591 (1993).

[17] A.P. Polychronakos, Phys. Rev. Lett. 69, 703 (1992).

[18] L. Brink, T.H. Hansson and M.A. Vasiliev, Phys. Lett. B286, 109 (1992).

[19] S.M. Girvin and T. Jach, Phys. Rev. B29, 5617 (1984).

[20] F.D.M. Haldane Phys. Rev. Lett. 51, 605 (1983).

[21] S.A. Trugman and S.A. Kivelson Phys. Rev. B26, 3682 (1985).

[22] J.K. Jain, Adv. Phys. 41, 105 (1992).

[23] S. Iso, D. Karabali and B. Sakita, Phys. Lett. B296, 143 (1992).

[24] S. Ouvry, Phys. Rev. D50, 5296 (1994).

[25] I.S. Gradshteyn and I.M. Ryzhik Table of integrals, series and products, Academic Press, 1980. 


\section{Figures}

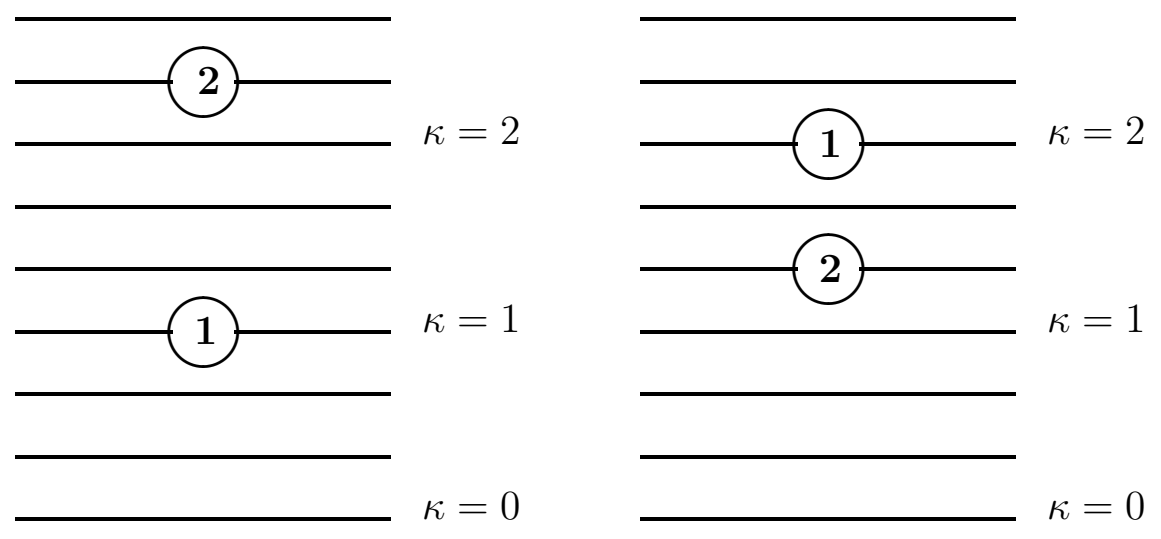

Figure 1. Two equivalent states of two particles in the $\nu=1 / 3$ system. The left one corresponds to the normal ordering prescription (3.25). 


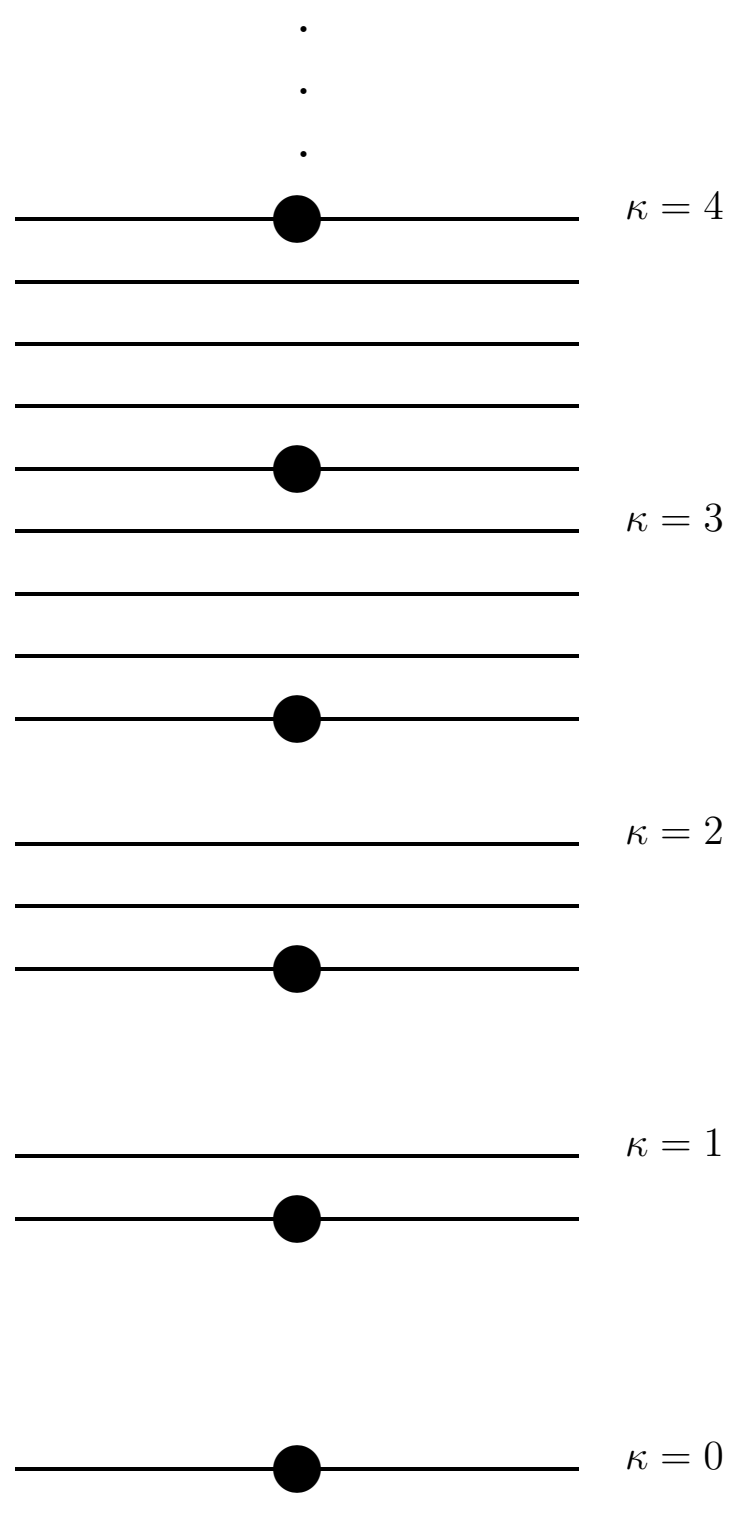

Figure 2. The ground state of the $\nu=4 / 5$ system. 